\title{
Photocurrent analysis of quantum cascade detectors by magnetotransport
}

\author{
François-Régis Jasnot, ${ }^{1}$ Nicolas Péré-Laperne, ${ }^{1}$ Louis-Anne de Vaulchier,${ }^{1, *}$ Yves Guldner, ${ }^{1}$ Francesca Carosella, ${ }^{1}$ \\ Robson Ferreira, ${ }_{1}^{1}$ Amandine Buffaz, ${ }^{2}$ Laetitia Doyennette, ${ }^{2}$ Vincent Berger, ${ }^{2}$ Mathieu Carras, ${ }^{3}$ and Xavier Marcadet ${ }^{3}$ \\ ${ }^{1}$ Laboratoire Pierre Aigrain, Ecole Normale Supérieure, CNRS (UMR 8551), \\ Université P. et M. Curie, Université Paris Diderot, 24 rue Lhomond, 75231 Paris Cedex 05, France \\ ${ }^{2}$ Laboratoire Matériaux et Phénomènes Quantiques, Université Paris Diderot - Paris 7 , \\ CNRS - UMR7162, Bâtiment Condorcet, 75205 Paris Cedex 13, France \\ ${ }^{3}$ Alcatel-Thales 3-5 lab, Route départementale 128, 91767 Palaiseau Cedex, France
}

(Dated: September 1, 2010)

\begin{abstract}
Photocurrent measurements have been performed on a quantum cascade detector structure under strong magnetic field $B$ applied parallel to the growth axis. The photocurrent shows oscillations as a function of $B$. In order to describe this behavior, we have developed a rate equation model. The interpretation of the experimental data supports the idea that an elastic scattering contribution plays a central role in the behavior of these structures. We present a calculation of the electron lifetime versus magnetic field which suggests that impurities scattering in the active region is the limiting factor. These experiments lead to a better understanding of these complex structures and identify key parameters to optimize them further.
\end{abstract}

\section{INTRODUCTION}

The quantum cascade detector, ${ }^{1}$ (QCD) recently proposed and realized in both the mid-infrared ${ }^{2}$ and in the $\mathrm{THz}^{3,4}$ range, is a photovoltaic version of the quantum well infrared photodetector (QWIP). ${ }^{5}$ The band structure of these devices is designed as a quantum cascade laser (QCL) under no applied electric field. ${ }^{1,3}$ As such, the QCD structure is designed to generate an electronic displacement under illumination through a cascade of quantum levels without the need of an applied voltage. They are totally passive systems and show a response only to photon excitation. Owing to this photovoltaic behavior, QCDs can work with a higher doping level than QWIPs and therefore possess higher quantum efficiencies, lower dark currents and longer integration times.

QCDs have already been realised with different material systems over the last few years. ${ }^{2,6-8}$ A typical mid-infrared QCD structure contains several identical periods, each one of them containing between 5 and 10 coupled quantum wells. A period is made of an 'active region' dedicated to the absorption of infrared photons and a 'cascade region' optimized for the electron transfer between two consecutive regions.

In a semiconductor quantum well structure, a magnetic field applied along the growth direction breaks the 2D in-plane continuum into discrete Landau levels (LLs). This experimental technique has been used to evaluate the different contributions of various scattering mechanisms in complex quantum cascade structures. ${ }^{4,9-12}$ The aim of the current magnetophotocurrent study is to understand electronic transport in QCDs under illumination and to study the scattering mechanisms involved in these complex structures at their working temperature $(80 \mathrm{~K})$. We further develop a simple model of transport under illumination in a QCD as well as calculations of rates for various electron scattering mechanisms. Through a comparison between experimental and calculation results, we highlight the mechanism limiting the response of QCDs.

\section{QCD STRUCTURE}

The QCD under study is a GaAs/AlGaAs heterostructure with a detection wavelength of $8 \mu \mathrm{m}$. It consists of 40 identical periods of 7 coupled GaAs quantum wells (QWs). $\quad \mathrm{Al}_{0.34} \mathrm{Ga}_{0.66} \mathrm{As}$ barriers are used in order to reach a conduction band-offset of $275 \mathrm{meV}$. N-doping of the first QW $\left(5 \times 10^{11} \mathrm{~cm}^{-2}\right)$ of each period allows to populate its first energy level $\mid$ down $\rangle$ in the conduction band with electrons (see Fig 1). We stress the fact that such doping level is much higher than in standard QCL structures, and also that dopants are placed in the first $\mathrm{QW}$, i.e., in the active region of the QCD. The layer sequence in $\AA$ starting from the first quantum well is as follows $67.8 / \mathbf{5 6 . 5} / 19.8 / \mathbf{3 9 . 6} / 22.6 / \mathbf{3 1 . 1} / 28.3 / \mathbf{3 1 . 1} / 33.9 / \mathbf{3 1 . 1} / 39.6 / \mathbf{3 1 . 1} / 4$ (the barrier widths are represented in bold types). Figure 1 recalls the principle of the device: owing to the absorption of a mid-infrared photon, an electron is excited from the fundamental level of the structure $\mid$ down $\rangle$ to the upper levels $|u p\rangle$ which are delocalized across the first two QWs. High dipole matrix elements between $|u p\rangle$ and the other energy levels of the cascade allows electrons to be transferred to the right QWs as a result of a series of LO-phonon scattering events. Levels in the cascade are labelled $\left|c_{i}\right\rangle$ with $i=1$ to 5 . The last QW of the cascade is identical to the first one and the period is repeated in order to increase the induced potential that results from this electron transfer. By closing the circuit, a significant photocurrent is expected without any applied bias. The studied samples are $100 \times 100 \mu \mathrm{m}^{2}$ square mesas obtained by reactive ion etching.

\section{MAGNETO-PHOTOCURRENT MEASUREMENTS}

QCDs are mounted inside an insert at the center of a superconducting coil where a magnetic field $B$ up to $16 \mathrm{~T}$ can be applied parallel to the growth axis. Light is emitted by a globar source from a Fourier transform infrared spectrometer and guided to the sample. The experiment consists in measur- 


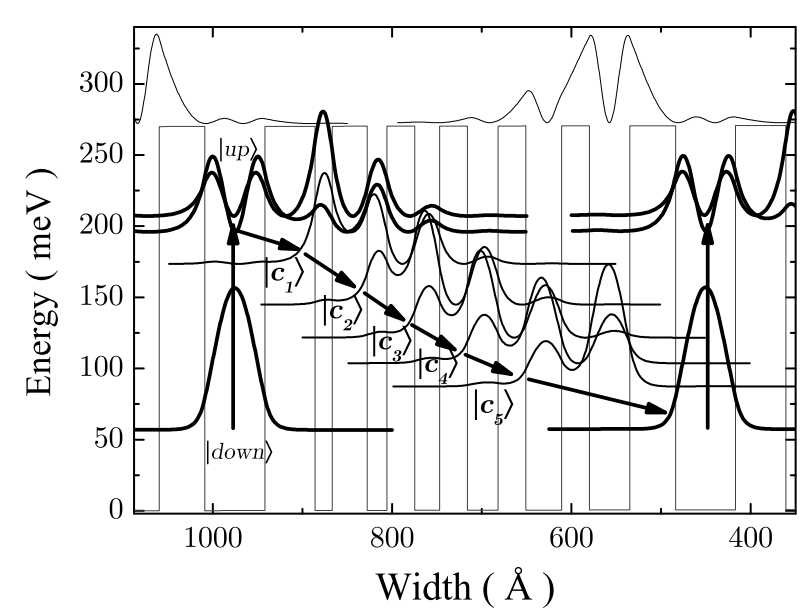

FIG. 1: Conduction band diagram of one period of an $8 \mu \mathrm{m}$ QCD showing the wavefunction of each energy levels. Note that the ground state of the first QW belongs to the former period and is noted $\mid$ down $\rangle$. The arrows illustrate the electronic path during a detection event.

ing the current under illumination $I_{\text {light }}$, without any applied voltage, at $80 \mathrm{~K}$ while the magnetic field is swept from zero to its maximum value.

Typical results are shown in figure 2(a). The photocurrent shows oscillations as a function of the magnetic field, superposed on a general behavior corresponding approximately to a quadratic decrease. This main decreasing component is attributed to the magneto-resistance of the contacts of the sample. ${ }^{12}$ This quadratic decrease has been removed from the experimental data in figure 2(b).

At zero magnetic field, all the quantum levels of a period have plane-wave-like energy dispersion in the direction parallel to the layers. At $0 \mathrm{~V}$ and without any illumination, electronic transitions from one level to another compensate each other resulting in zero current (the system is in equilibrium). ${ }^{13}$ When a magnetic field is applied along the growth axis, the subbands split into ladders of discrete Landau levels given by:

$$
E_{n, p}=E_{n}^{0}+\left(p+\frac{1}{2}\right) \hbar \omega_{c}
$$

where $n$ and $p$ are integers, $n$ is the index of the subband and $p$ the index of the Landau level, $\hbar \omega_{c}=\hbar e B / m^{\star}$ is the cyclotron energy, $e$ is the electronic charge and $m^{\star}$ the effective mass in GaAs. $E_{n}^{0}$ is the energy of the subband edge at zero magnetic field. The effect of the magnetic field on the QCD photocurrent is quite similar to that in a three-level active region of a QCL where electron-scattering from the upper state is modulated by the magnetic field. ${ }^{9,10}$ Indeed, depending on the value of the magnetic field, the Landau level arrangement strongly influences the scattering of electrons from the various $|u p, 0\rangle$ levels to $|n, p\rangle$, where $|n, p\rangle$ designates the $p$ Landau level originating from subband

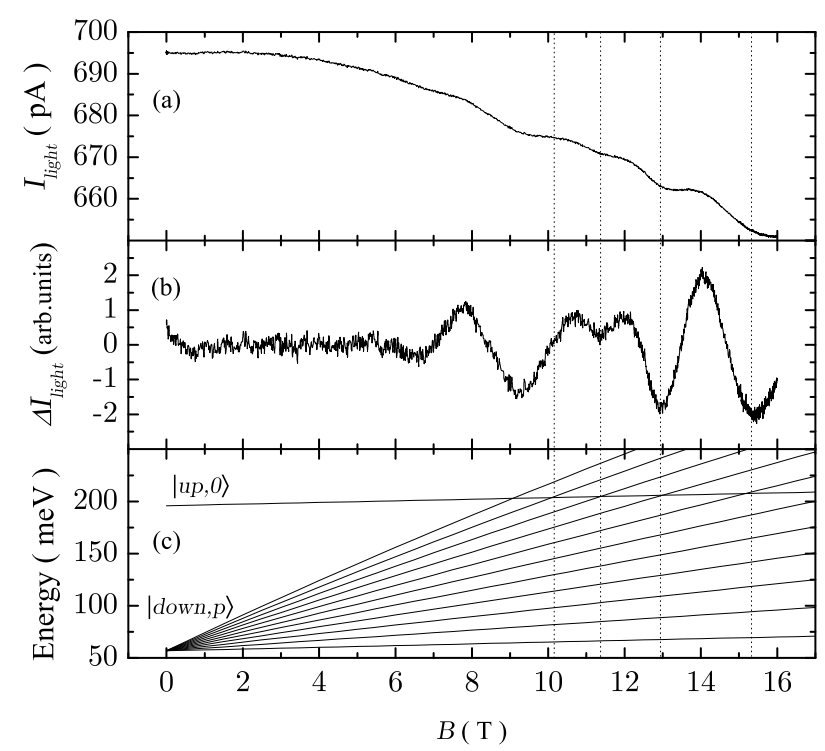

FIG. 2: (a) Current under illumination as a function of magnetic field at zero bias. (b) $I_{\text {light }}$ as a function of the magnetic field where the contribution of the magnetoresistance has been subtracted. (c) Fanchart of $|u p, 0\rangle$ and $\mid$ down, $p\rangle$ as a function of $B$ from Eq. 1 taking into account the band nonparabolicity.

$n$.

Figure 2(c) represents the evolution in energy of the Landau levels $|u p, 0\rangle$ and $\mid$ down, $p\rangle$ as a function of magnetic field from Eq. 1, taking into account the band nonparabolicity. Minima of current in magnetic field are in good agreement with crossing of LL $|u p, 0\rangle$ with LLs $\mid$ down, $p\rangle$ with $p=6,7,8,9$ at $B=15.3 \mathrm{~T}, 13.0 \mathrm{~T}, 11.4 \mathrm{~T}, 10.1 \mathrm{~T}$, respectively (dashed vertical bars in Fig. 2). Comparing Figure 2(b) and 2(c) leads to the conclusion that an elastic scattering mechanism is dominant in this structure and mainly involves $|u p\rangle$ and $\mid$ down $\rangle$ levels. A complete description of the mechanisms involved in the photocurrent and a model describing it as a function of $B$ are presented in parts IV and V. Oscillations at low magnetic field, between $B=4 \mathrm{~T}$ and $9 \mathrm{~T}$ are described in part V.

\section{MODEL AND SCATTERING MECHANISMS INVOLVED IN THE CURRENT}

In a previous paper, we presented magneto-current measurement without any illumination. ${ }^{12}$ These experiments put in evidence some leakages from level $\mid$ down $\rangle$ to the levels in the next cascade when the detector is submitted to an applied bias. This current is called the dark current $\left(I_{\text {dark }}\right)$ and we demonstrated $I_{\text {dark }}$ originates from several parallel cross transitions for a fixed temperature. As described in part III, we do not apply any bias on our structure for these photocurrent experiments: the current measured in this work 
originates from a displacement of electrons after a photon absorption.

In this part, we describe in detail the model that leads to the magneto-photocurrent oscillations in a QCD. We propose a model of transport within one single period by a rate equation approach. We assume that electrons are in the upper detector state $(|u p\rangle)$ through absorption of a photon and we establish the rate equation for this level. Electrons can leave this state either by falling back to the fundamental level $\mid$ down $\rangle$ or through the cascade $\left|c_{i}\right\rangle$. i.e.:

$\frac{\mathrm{d} N_{u p}}{\mathrm{~d} t}=\frac{N_{\text {down }}}{\tau_{\text {down }- \text { up }}}-\frac{N_{u p}}{\tau_{\text {up }- \text { down }}}+\frac{N_{c}}{\tau_{c-u p}}-\frac{N_{u p}}{\tau_{u p-c}}+\alpha N_{\text {down }}$

where the subscribe $c$ stands for the whole cascade, $N_{i}$ is the sheet density of level $|i\rangle, \frac{1}{\tau_{u p-c}}=\sum_{i=1}^{5} \frac{1}{\tau_{u p-c_{i}}}, \tau_{m-n}$ the scattering time of an electron in level $|m\rangle$ towards the level $|n\rangle$ and $\alpha$ the absorption efficiency coefficient. The current we are measuring in our macroscopic experimental setup is given by:

$$
\frac{J}{q}=\frac{N_{u p}}{\tau_{u p-c}}-\frac{N_{c}}{\tau_{c-u p}}
$$

where $J$ is the current density and $q$ the charge of electron. We recall the equilibrium conditions explained in part III when no bias is applied and without illumination:

$$
\left\{\begin{array}{l}
\frac{N_{\text {down }}^{e}}{\tau_{\text {down-up }}}=\frac{N_{u p}^{e}}{\tau_{u p-\text { down }}} \\
\frac{N_{u p}^{e}}{\tau_{u p-c}}=\frac{N_{c}^{e}}{\tau_{c-u p}}
\end{array}\right.
$$

The exponent ' $e$ ' signifies equilibrium. We assume that the population of level $\mid$ down $\rangle$ is the same in equilibrium and under illumination $\left(N_{\text {down }} \approx N_{\text {down }}^{e}\right)$ and obviously the population of level $|u p\rangle$ varies under illumination $\left(N_{u p} \neq N_{u p}^{e}\right)$.

In a stationary state we find from equations 2 and 3:

$$
\frac{J}{q}=\frac{N_{u p}^{e}}{\tau_{u p-\text { down }}}-\frac{N_{u p}}{\tau_{\text {up-down }}}+\alpha N_{\text {down }}
$$

The variation of population of level $|u p\rangle$ in the two different situations can be expressed as:

$$
N_{u p}-N_{u p}^{e}=\alpha N_{\text {down }} \tau_{u p}
$$

where $\frac{1}{\tau_{u p}}=\frac{1}{\tau_{u p-\text { down }}}+\frac{1}{\tau_{u p-c}}$
As a conclusion the current is given by:

$$
\frac{J}{q}=\alpha N_{\text {down }}\left(\frac{\tau_{\text {up-down }}}{\tau_{\text {up }- \text { down }}+\tau_{u p-c}}\right)=\alpha N_{\text {down }} Q E
$$

As mentioned above, as $\alpha$ and $N_{\text {down }}$ are constant, the only figure which varies under magnetic field is the quantum efficiency QE, as a function of the subband lifetimes.

This model is in agreement with the trade-off presented in Ref. 12, where the efficiency of the detector is ruled by the ability to generate photocarriers and then to extract them owing to the cascade. As a consequence, both lifetimes $\tau_{u p-d o w n}$ and $\tau_{u p-c}$ have to be involved in the photocurrent.

The lifetimes are directly obtained from calculation of the scattering rates of the different elastic and inelastic mechanisms. As mentioned in Ref. 14 and Ref. 10, two mechanisms are dominant in these mid-infrared GaAs cascade structures LO-phonon emission and interface roughness.

We present in table I the calculated scattering rates of the different processes at $B=0 \mathrm{~T}$. For interface roughness, we used a Gaussian autocorrelation of the roughness, with an average height of $\Delta=2.8 \AA$ and a correlation length of $\Lambda=60 \AA^{15}$ LO phonon emission scattering time has been calculated as in Ref. 14. In our structure, a third scattering mechanism becomes important - ionized impurities scattering. In Ref. 10 this process has been neglected as the doped layers were separated from the optical transition. In this work, instead, the doping level is much higher and the doped layers is in the core of the active region. As a direct consequence of these two effects, we calculate that impurities scattering is the most efficient process in our system.

\begin{tabular}{ccc}
\hline Scattering mechanism & $1 / \tau_{u p-\text { down }}$ & $1 / \tau_{u p-c}$ \\
\hline LO phonon emission & $7.0 \times 10^{11}$ & $7.9 \times 10^{11}$ \\
Interface roughness & $6.0 \times 10^{11}$ & $8.6 \times 10^{12}$ \\
Impurity scattering & $1.8 \times 10^{13}$ & $5.2 \times 10^{13}$ \\
\hline
\end{tabular}

TABLE I: Scattering rates in $\mathrm{s}^{-1}$ are calculated using different scattering processes for an electron in the $|u p\rangle$ subband at $B=0 \mathrm{~T}$.

In order to take into account the main scattering process we calculate ionized-impurities scattering as a function of magnetic field. The electron-ionized impurity interaction potential is given by ${ }^{16}$ :

$$
V_{\mathrm{imp}}\left(\mathbf{R}, \mathbf{R}_{i}\right)=\frac{2 \pi e^{2}}{4 \pi \varepsilon_{0} \varepsilon_{r} S} \sum_{\mathbf{Q}_{\perp}} \frac{1}{Q_{\perp}} \exp \left[-Q_{\perp}\left|z-z_{i}\right|+\mathrm{i} \mathbf{Q}_{\perp} \cdot\left(\boldsymbol{\rho}-\boldsymbol{\rho}_{i}\right)\right]
$$


where $S=L_{x} L_{y}$ is the sample surface, $\mathbf{R}_{i}=\left(\boldsymbol{\rho}_{i}, z_{i}\right)$ is the impurity position in the well, and $\mathbf{Q}_{\perp}=\left(Q_{x}, Q_{y}\right)$ is the electron wavevector in the layer plane. The electron wavefunction, in presence of a magnetic field applied along the heterostructure growth direction, is given by

$$
\left|m, n, k_{y}\right\rangle=\chi_{m}(z) \phi_{n}\left(x+\ell_{c}^{2} k_{y}\right) \frac{\mathrm{e}^{\mathrm{i} k_{y} y}}{\sqrt{L_{y}}}
$$

where $\chi_{m}(z)$ is the heterostructure envelope function corresponding to the $\mathrm{m}^{\text {th }}$ subband, $\phi_{n}$ is the $\mathrm{n}^{\text {th }}$ Hermite function associated with the $\mathrm{n}^{\text {th }}$ Landau level and $\ell_{c}=\sqrt{\hbar / e B}$ is the magnetic length. We calculate the scattering time for an electron in the lowest Landau level $(n=0)$ of the upper subband towards the Landau levels of lower subband using Fermi's golden rule and assuming broadened Landau levels, ${ }^{14}$ giving:

$$
\left\langle\frac{1}{\tau_{2, n=0, k_{y}}}\right\rangle=\frac{2 \pi}{\hbar} \sum_{i} \sum_{n^{\prime}} \sum_{k_{y}^{\prime}}\left|\left\langle 1, n^{\prime}, k_{y}^{\prime}\left|V_{\mathrm{imp}}\left(\mathbf{R}, \mathbf{R}_{i}\right)\right| 2, n=0, k_{y}\right\rangle\right|^{2} \frac{1}{\delta \sqrt{2 \pi}} \exp \left[-\frac{\left(E_{2}-E_{1}-n^{\prime} \hbar \omega_{c}\right)^{2}}{2 \delta^{2}}\right]
$$

where $\delta$ is the broadening parameter of LLs. According to Ref. 14, the inhomogeneous broadening model results in an expression of the average scattering rate which resembles that obtained by replacing the deltalike peaks of the Landau levels density of states by Gaussian functions. As such we take $\delta=6 \mathrm{meV}$, a value consistent with $\hbar / \tau_{\text {up }}^{i m p}$ down obtained in the following. The calculation of the matrix element leads to

$$
\begin{aligned}
\left\langle\frac{1}{\tau_{2, n=0, k_{y}}}\right\rangle= & \frac{2 \pi}{\hbar}\left(\frac{e^{2}}{4 \pi \varepsilon_{0} \varepsilon_{r}}\right)^{2} N_{\mathrm{imp}_{S}} \\
& \times \sum_{z_{i}} \sum_{n^{\prime}} \iint \frac{\mathrm{d} Q_{x} \mathrm{~d} Q_{y}}{Q_{x}^{2}+Q_{y}^{2}}\left|\left\langle 1\left|\mathrm{e}^{-\sqrt{Q_{x}{ }^{2}+Q_{y}{ }^{2}}\left|z-z_{i}\right|}\right| 2\right\rangle\right|^{2}\left|\left\langle n^{\prime}, k_{y}+Q_{y}\left|\mathrm{e}^{\mathrm{i} Q_{x} x}\right| n=0, k_{y}\right\rangle\right|^{2} \\
& \times \frac{1}{\delta \sqrt{2 \pi}} \exp \left[-\frac{\left(E_{2}-E_{1}-n^{\prime} \hbar \omega_{c}\right)^{2}}{2 \delta^{2}}\right] .
\end{aligned}
$$

where $N_{\text {imp }_{S}}$ is the area impurity density. Making the variable change $\tilde{x}=x+\ell_{c}^{2} k_{y}$ and following Mycielski et al. ${ }^{17}$ we obtain

$$
\left\langle n^{\prime}, k_{y}+Q_{y}\left|\mathrm{e}^{\mathrm{i} Q_{x} x}\right| n=0, k_{y}\right\rangle=\mathrm{e}^{-\mathrm{i} Q_{x} \ell_{c}^{2} k_{y}} \frac{1}{\sqrt{n^{\prime} !}} \exp \left(-\frac{\mathrm{i}}{2} \ell_{c}^{2} Q_{x} Q_{y}\right)\left(\frac{\ell_{c}}{\sqrt{2}}\right)^{n^{\prime}}\left(Q_{y}+\mathrm{i} Q_{x}\right)^{n^{\prime}} \exp \left[-\frac{1}{4} \ell_{c}^{2}\left(Q_{x}^{2}+Q_{y}^{2}\right)\right]
$$

which introduced in the previous equation gives

$$
\begin{aligned}
& \left\langle\frac{1}{\tau_{2, n=0, k_{y}}}\right\rangle=\frac{2 \pi}{\hbar}\left(\frac{e^{2}}{4 \pi \varepsilon_{0} \varepsilon_{r}}\right)^{2} N_{\mathrm{imp}_{S}} \\
& \quad \times \sum_{z_{i}} \sum_{n^{\prime}} \frac{1}{n^{\prime} !}\left(\frac{\ell_{c}^{2}}{2}\right)^{n^{\prime}} \iint \mathrm{d} Q_{x} \mathrm{~d} Q_{y}\left(Q_{x}^{2}+Q_{y}^{2}\right)^{n^{\prime}-1} \exp \left[-\frac{\ell_{c}^{2}}{2}\left(Q_{x}^{2}+Q_{y}^{2}\right)\right]\left|\left\langle 1\left|\mathrm{e}^{-\sqrt{Q_{x}^{2}+Q_{y}{ }^{2}}\left|z-z_{i}\right|}\right| 2\right\rangle\right|^{2} \\
& \times \frac{1}{\delta \sqrt{2 \pi}} \exp \left[-\frac{\left(E_{2}-E_{1}-n^{\prime} \hbar \omega_{c}\right)^{2}}{2 \delta^{2}}\right] .
\end{aligned}
$$

Then using polar coordinates $\mathrm{d} Q_{x} \mathrm{~d} Q_{y}=Q_{\perp} \mathrm{d} Q_{\perp} \mathrm{d} \theta$ and making the variable change $X=\frac{\ell_{c}^{2}}{2} Q_{\perp}^{2}$, the final expression obtained is:

$$
\begin{aligned}
\left\langle\frac{1}{\tau_{2, n=0, k_{y}}}\right\rangle & =\frac{2 \pi^{2}}{\hbar}\left(\frac{e^{2}}{4 \pi \varepsilon_{0} \varepsilon_{r}}\right)^{2} N_{\mathrm{imp}_{S}} \\
& \times \sum_{z_{i}} \sum_{n^{\prime}} \frac{1}{\delta \sqrt{2 \pi}} \exp \left[-\frac{\left(E_{2}-E_{1}-n^{\prime} \hbar \omega_{c}\right)^{2}}{2 \delta^{2}}\right] \frac{1}{n^{\prime} !} \int_{0}^{+\infty} X^{n^{\prime}-1} \mathrm{e}^{-X}\left|\left\langle 1\left|\exp \left(-\frac{\sqrt{2 X}}{\ell_{c}}\left|z-z_{i}\right|\right)\right| 2\right\rangle\right|^{2} \mathrm{~d} X
\end{aligned}
$$

Figure 3 presents a comparison between the experimental 


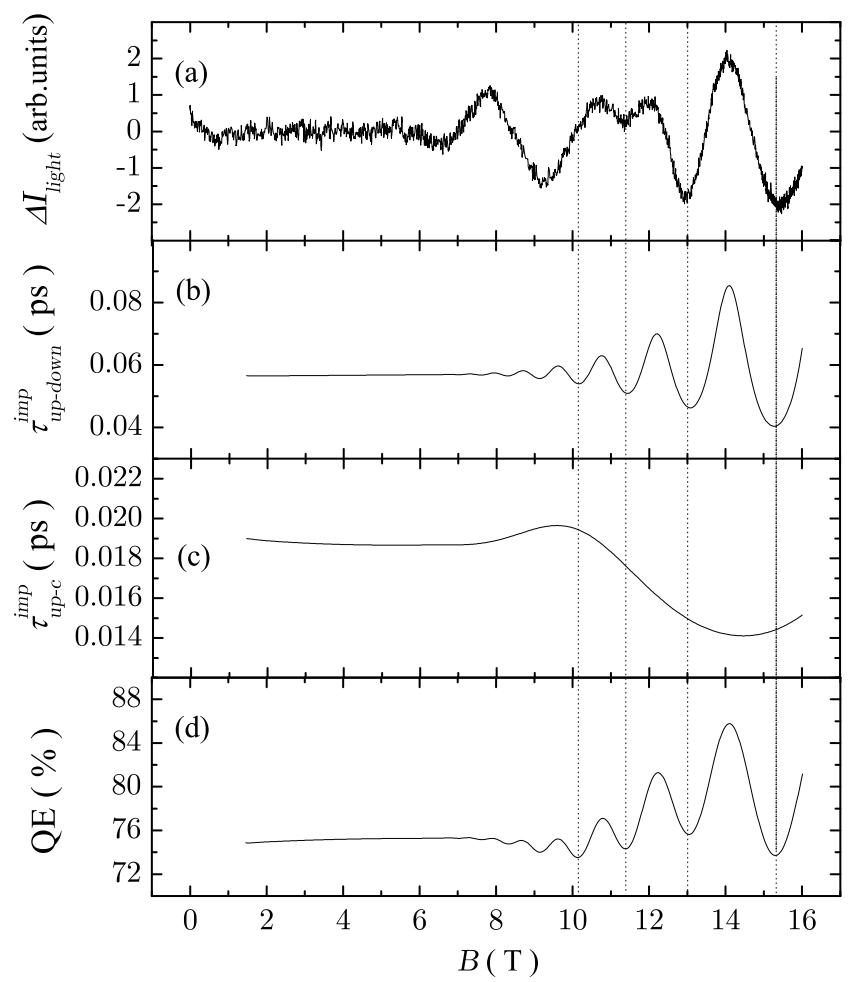

FIG. 3: (a) $\Delta I_{\text {light }}$ as a function of magnetic field where the contribution of the magneto-resistance has been subtracted. ${ }^{12}$ (b) Ionized impurity scattering $\tau_{u p-\text { down }}^{i m p}$ under magnetic field between $|u p\rangle$ and $\mid$ down $\rangle$ levels. (c) Ionized impurity scattering $\tau_{u p-c}^{i m p}$ under magnetic field between $|u p\rangle$ and levels in the cascade. (d) Quantum efficiency $(Q E)$ calculated with Eq. 7

times as a function of magnetic field. Minima in photocurrent at high field correspond to minima in the calculation (vertical dotted lines) and the photocurrent behavior is attributed to oscillations of $\tau_{u p-d o w n}^{i m p}$.

\section{INTERPRETATION OF EXPERIMENTAL DATA}

In this section, we concentrate on the description of photocurrent as a function of $B$ owing to Eq. 7. Low magnetic field photocurrent oscillations are also discussed. Figure 3 presents a comparison between experimental results and our model. Figures 3(b) and 3(c) show the two scattering times involved in Eq. 7 as a function of $B$ calculated with electron-ionized impurities scattering. Figure 3(d) shows the calculation of the related quantum efficiency.

First, the oscillating behavior at high magnetic field $(B>9 \mathrm{~T})$ originates from the scattering process involved in the electronic transfer from $|u p\rangle$ to $\mid$ down $\rangle$. This transfer leads to minima in the current which fit well with $\tau_{u p-d o w n}^{i m p}$ and the $Q E$. At the same time $\tau_{u p-c}^{i m p}$ has a long period oscillating behavior as a function of $B$ enhancing the peak in $Q E$ at $B=14 \mathrm{~T}$ in accordance with experimental data. $Q E$ is the most relevant figure and describes the performance of the detector. Under magnetic field, the $Q E$ oscillates between $74 \%$ and $85 \%$. Note that, on one hand, ionized impurities scattering is a limiting factor within $\tau_{u p-d o w n}^{i m p}$ but, on the other hand, is an enhancing factor for $\tau_{u p-c}^{i m p}$. By extrapolating the data under magnetic field, at $B=0 \mathrm{~T}, Q E$ is equal to $75 \%$, a value that should be increased to improve the detector performance. An optimized structure should take these results into account by shifting the ionized impurities from the active region, where they are enhancing $1 / \tau_{u p-d o w n}^{i m p}$, to a position where they would only contribute to $\tau_{u p-c}^{i m p}$.

Second, at low magnetic field $(B<9 \mathrm{~T})$, there is a discrepancy between the experimental oscillating behavior and the calculed $Q E$, especially at $8 \mathrm{~T}$ where the data show a broad peak. There can be two reasons for this: (i) the calculation of $\tau_{u p-c}$ itself, or (ii) a scattering mechanism for the transport of electrons away from the active region, inside the cascade. Concerning the first one, in the calculation of $\tau_{u p-c}$ we took into account the impurity scattering mechanism which is found to be stronger than any other mechanism, in particular LO-phonon emission. This is due to the fact that the energy separation between the $|u p\rangle$ and $\left|c_{1}\right\rangle$ states is short by $\sim 13 \mathrm{meV}$ to the LO-phonon energy. But there exists a particular magnetic field at which the first Laudau level of $|u p\rangle$ will be separated from $\left|c_{1}\right\rangle$ by exactly one LO-phonon. This should occur at 13/1.6 $8 \mathrm{~T}$ and would enhance greatly the extraction of electrons from the active region to the cascade. Concerning the second mechanism that can enhance the peak at $8 \mathrm{~T}$, it can be seen in Fig. $2 \mathrm{~b}$ that $\Delta I_{\text {light }}$ presents actually two maxima at $B=5.8 \mathrm{~T}$ and $B=8 \mathrm{~T}$. According to Eq.1, the characteristic energy of this series of oscillations is $\Delta E \sim 37 \mathrm{meV}$. This energy is close to the separation energy of subsequent levels in the cascade. In the vicinity of the crossings of $\left|c_{i}, 0\right\rangle$ and $\left|c_{i+1}, p\right\rangle$, interface roughness scattering enhances the flow of electrons through the cascade. Assuming that this series is relevant for our structure, two extra maxima should arise at $B=11.7 \mathrm{~T}$ and $B=23.4 \mathrm{~T}$. The latter is out of our experimental range, but the former is present on $\Delta I_{\text {light }}$ curve superimposed on the short period oscillating behavior of $\tau_{u p-\text { down }}^{i m p}$. As such, this new series of resonances in the cascade region also provides an explanation to the discrepancy in amplitude between the experimental results and the calculation of $Q E$ around $12 \mathrm{~T}$.

Previously, two models were developed for this structure to describe the dark current. ${ }^{12,18}$ The first one assumed a quasi-Fermi equilibrium on all the structure due to a fast extraction in the cascade whereas the second one assumed a thermalized population in each subband of the cascade. This second model helped to describe the dark current at high temperatures, and pointed out the cascade design as a crucial step in the structure definition. Thanks to the present work, extraction towards the cascade as well as intra-cascade resonances are highlighted as optimizing factors which have 
to be improved to increase the performance of the detector.

Finally, it is worth stressing that the physics in this particular QCD is quite different from the one in usual quantum cascade structures (for example quantum cascade lasers) because the doping level is much higher and positioned in the active absorption layer. As a consequence, usual scattering interactions are calculated to be less efficient than the impurity scattering as shown in table I. For instance in Ref. 3, the doping level corresponds to an effective sheet carrier density of $8.7 \times 10^{9} \mathrm{~cm}^{-2}$ instead of $5 \times 10^{11} \mathrm{~cm}^{-2}$ in our sample. As such, there is no contradiction with former work in quantum cascade structures.

\section{CONCLUSION}

In conclusion, we have investigated the photocurrent in a mid-infrared quantum cascade detector as a function of magnetic field. We have described the high magnetic field oscillations by an elastic scattering mechanism, namely electron-ionized impurities scattering, which is found to be the dominant scattering mechanism in our sample. We have also developed a model to describe the photocurrent in this structure. It highlights the trade-off in transport in this structure involving both a limiting scattering time $\tau_{u p-\text { down }}^{i m p}$ and an enhancing scattering time $\tau_{u p-c}^{i m p}$. We have performed calculations in order to describe electron-impurities scattering as a function of magnetic field and to evaluate $\tau_{u p-d o w n}^{i m p}$ and $\tau_{u p-c}^{i m p}$. Finally, we have used both our model and calculations to define and evaluate the quantum efficiency of the structure. In order to improve further this efficiency, we suggest to shift the impurities in another location of the structure in order to minimize $1 / \tau_{\text {up - down }}^{i m p}$. Moreover magnetic field has allowed us to highlight the crucial part of the design of the cascade in order to enhance $\tau_{u p-c}^{i m p}$. Thanks to this work we assess the different fundamental electronic mechanisms within these complex heterostructures.

\section{ACKNOWLEDGMENTS}

The Laboratoire Pierre Aigrain is a "Unite Mixte de Recherche" (UMR 8551) between Ecole Normale Supérieure, the CNRS, the University Pierre et Marie Curie (Paris 6) and the University Paris Diderot - Paris 7). This work has been supported by a grant of the Agence Nationale pour la Recherche (ANR BLANC 0257). We would like to thank G. Bastard and S. Dhillon for very valuable and fruitful discussions.
* Electronic address: louis-anne.devaulchier@lpa. ens.fr

${ }^{1}$ L. Gendron, M. Carras, A. Huynh, V. Ortiz, C. Koeniguer, and V. Berger, Appl. Phys. Lett. 85, 2824 (2004).

${ }^{2}$ L. Gendron, C. Koeniguer, V. Berger, and X. Marcadet, Appl. Phys. Lett. 86, 121116 (2005).

${ }^{3}$ M. Graf, G. Scalari, D. Hofstetter, J. Faist, H. Beere, E. Linfield, D. Ritchie, and G. Davies, Appl. Phys. Lett. 84, 475 (2004).

${ }^{4}$ G. Scalari, M. Graf, D. Hofstetter, J. Faist, H. Beere, and D. Ritchie, Semicond. Sci. Technol. 21, 1743 (2006).

5 B.F. Levine, K.K. Choi, C.G. Bethea, J. Walker, and R.J. Malik, Appl. Phys. Lett. 50, 1092 (1987).

${ }^{6}$ M. Graf, N. Hoyler, M. Giovannini, J. Faist, and D. Hofstetter, Appl. Phys. Lett. 88, 241118 (2006).

7 F.R. Giorgetta, E. Baumann, M. Graf, L. Ajili, N. Hoyler, M. Giovannini, J. Faist, and D. Hofstetter, Appl. Phys. Lett. 90, 231111 (2007).

8 A. Buffaz, M. Carras, L. Doyennette, A. Nedelcu, X. Marcadet, and V. Berger, Appl. Phys. Lett. 96, 172101 (2010).

9 D. Smirnov, O. Drachenko, J. Leotin, H. Page, C. Becker, C. Sirtori, V. Apalkov, and T. Chakraborty, Phys. Rev. B 66, 125317
(2002).

10 A. Leuliet, A. Vasanelli, A. Wade, G. Fedorov, D. Smirnov, G. Bastard, and C. Sirtori, Phys. Rev. B 73, 085311 (2006).

11 N. Péré-Laperne, L.A. de Vaulchier, Y. Guldner, G. Bastard, G. Scalari, M. Giovannini, J. Faist, A. Vasanelli, S. Dhillon, and C. Sirtori, Appl. Phys. Lett. 91, 062102 (2007).

12 A. Gomez, N. Péré-Laperne, L.A. de Vaulchier, C. Koeniguer, A. Vasanelli, A. Nedelcu, X. Marcadet, Y. Guldner, and V. Berger, Phys. Rev. B 77, 085307 (2008).

13 C. Koeniguer, G. Dubois, A. Gomez, and V. Berger, Phys. Rev. B 74, 235325 (2006)

${ }^{14}$ C. Becker, A. Vasanelli, C. Sirtori, and G. Bastard, Phys. Rev. B 69, 115328 (2004)

15 H. Sakaki, T. Noda, K. Hirakawa, M. Tanaka, and T. Matsusue, Appl. Phys. Lett. 51, 1934 (1987).

16 R. Ferreira, and G. Bastard, Phys. Rev. B 40, 1074 (1989).

17 J. Mycielski, G. Bastard, and C. Rigaux, Phys. Rev. B 16, 1675 (1977).

18 A. Buffaz, A. Gomez, M. Carras, L. Doyennette, and V. Berger, Phys. Rev. B 81, 075304 (2010). 